\title{
Multisensory Temporal Integration in Autism Spectrum Disorders
}

\author{
Ryan A. Stevenson, ${ }^{1,2,3,4}$ Justin K. Siemann, ${ }^{2,3,5}$ Brittany C. Schneider, ${ }^{6}$ Haley E. Eberly, ${ }^{6}$ Tiffany G. Woynaroski, ${ }^{1}$ \\ Stephen M. Camarata, ${ }^{3,4}$ and Mark T. Wallace ${ }^{1,2,3,7,8}$ \\ ${ }^{1}$ Department of Hearing and Speech Sciences, Vanderbilt University Medical Center, Nashville, Tennessee 37232, ${ }^{2}$ Vanderbilt Brain Institute, Nashville, \\ Tennessee 37232, ${ }^{3}$ Vanderbilt Kennedy Center, Nashville, Tennessee 37203, ${ }^{4}$ Department of Psychology, University of Toronto, Toronto, Ontario M5S 3G3, \\ Canada, ${ }^{5}$ Neuroscience Graduate Program, Vanderbilt University Medical Center, Nashville, Tennessee 37232, ${ }^{6}$ Program in Neuroscience, Vanderbilt \\ University, Nashville, Tennessee 37232, ${ }^{7}$ Department of Psychology, Vanderbilt University, Nashville, Tennessee 37232, and ${ }^{8}$ Department of Psychiatry, \\ Vanderbilt University, Nashville, Tennessee 37232
}

The new DSM-5 diagnostic criteria for autism spectrum disorders (ASDs) include sensory disturbances in addition to the well-established language, communication, and social deficits. One sensory disturbance seen in ASD is an impaired ability to integrate multisensory information into a unified percept. This may arise from an underlying impairment in which individuals with ASD have difficulty perceiving the temporal relationship between cross-modal inputs, an important cue for multisensory integration. Such impairments in multisensory processing may cascade into higher-level deficits, impairing day-to-day functioning on tasks, such as speech perception. To investigate multisensory temporal processing deficits in ASD and their links to speech processing, the current study mapped performance on a number of multisensory temporal tasks (with both simple and complex stimuli) onto the ability of individuals with ASD to perceptually bind audiovisual speech signals. High-functioning children with ASD were compared with a group of typically developing children. Performance on the multisensory temporal tasks varied with stimulus complexity for both groups; less precise temporal processing was observed with increasing stimulus complexity. Notably, individuals with ASD showed a speech-specific deficit in multisensory temporal processing. Most importantly, the strength of perceptual binding of audiovisual speech observed in individuals with ASD was strongly related to their low-level multisensory temporal processing abilities. Collectively, the results represent the first to illustrate links between multisensory temporal function and speech processing in ASD, strongly suggesting that deficits in low-level sensory processing may cascade into higher-order domains, such as language and communication.

Key words: audiovisual; autism spectrum disorders; multisensory integration; sensory processing; speech perception; temporal processing

\section{Introduction}

Autism spectrum disorders (ASDs) are associated with atypical communication, deficits in social reciprocity, and restricted interests/repetitive behaviors. Individuals with ASD also exhibit atypical sensory processing. Since Kanner's original description of autism, which references a number of sensory disturbances (Kanner, 1943), atypical sensory processing has been reported in a number of different sensory modalities (for review, see Marco et al., 2011). Much of this evidence has come through caregiver

\footnotetext{
Received Aug. 23, 2013; revised Nov. 8, 2013; accepted Nov. 13, 2013.

Author contributions: R.A.S., S.M.C., and M.T.W. designed research; R.A.S., J.K.S., B.C.S., and H.E.E. performed research; R.A.S., J.K.S., T.G.W., S.M.C., and M.T.W. contributed unpublished reagents/analytic tools; R.A.S. and J.K.S. analyzed data; R.A.S., J.K.S., T.G.W., and M.T.W. wrote the paper.

This work was supported by National Institutes of Health Grant R34 DC010927, Evaluation of Sensory Integration Treatment in ASD, National Institutes of Health Grant F32 DC011993, National Institutes of Health Grant R21 CA183492, Multisensory Integration and Temporal Processing in ASD, Simons Foundation Explorer Award, Vanderbilt Kennedy Center MARI/Hobbs Award, and the Vanderbilt Brain Institute. We thank Zachary Barnett for technical assistance and Lena Quinto for the speech stimuli.

The authors declare no competing financial interests.

Correspondence should be addressed to Dr. Ryan A. Stevenson, 465 21st Avenue South, 7110 MRB III BioSci BIdg, Nashville, TN 37232. E-mail: ryan.andrew.stevenson@gmail.com.

DOI:10.1523/JNEUROSCI.3615-13.2014

Copyright $\odot 2014$ the authors $\quad 0270-6474 / 14 / 340691-07 \$ 15.00 / 0$
}

reports and questionnaires, with a relative paucity of empirical work examining sensory function in ASD.

One consistent finding (though not universal) is unimpaired or enhanced processing of simple sensory stimuli contrasted against impaired processing of complex stimuli (e.g., Bertone et al., 2003). Such evidence supports several of the more prominent theories of autism, including weak central coherence, which proposes that a core difference in ASD is the ability to combine pieces of information into a unified perceptual whole (Frith and Happe, 1994). One example of this is the "binding" of information from multiple sensory modalities that specify a single object or event, such as that seen with the auditory and visual components of a speech signal. Multisensory binding and integration convey a host of behavioral gains, including substantial benefits in speech comprehension (Sumby and Pollack, 1954). Recently, research has revealed changes in the manner in which individuals with ASDs combine information across the different sensory modalities. One example of this altered multisensory processing is a reduced susceptibility to the McGurk effect (Gelder et al., 1991; Smith and Bennetto, 2007), where pairing discordant visual and auditory speech-related cues typically results in reports of a novel percept reflecting the synthesis of the two sensory channels 
(McGurk and MacDonald, 1976). Individuals with ASDs also show less efficient integration with non-language stimuli, both behaviorally and neurally (Brandwein et al., 2013).

Many factors impact how sensory signals are integrated across the different modalities, with one of the strongest of these being the temporal relationship between the sensory inputs. Thus, the behavioral and perceptual gains derived from multisensory binding depend upon the temporal relationship of the paired stimuli, with gains typically being observed only within a "window" of temporal offsets between inputs. Impairments in temporal processing are well documented in ASDs (Brock et al., 2002; Oram Cardy et al., 2005), including impairments in multisensory temporal function, suggesting that individuals with ASDs are more likely to perceive highly asynchronous stimuli as synchronous relative to typically developing (TD) peers (Foss-Feig et al., 2010; Kwakye et al., 2011). However, despite evidence for deficits in both temporal processing and audiovisual perceptual binding, the relationship between them in ASDs remains unclear. Work in TD populations has shown that individuals with lower multisensory temporal acuity (and thus the less reliable temporal synchrony is as a cue to determine which sensory inputs should be perceptually bound) exhibit weaker integration (Stevenson et al., 2012). Here, we explored the hypothesis that alterations in multisensory temporal processing may be related to deficits in audiovisual speech integration in individuals with ASDs.

\section{Materials and Methods}

Participants. Participants included 32 ASD and 32 TD age-matched individuals from 6 to 18 years old $(\mathrm{ASD}=11.8 \pm 3.2, \mathrm{TD}=12.3 \pm 2.3)$, and matched on the matrix reasoning subtest Wechsler Abbreviated Scale of Intelligence, Second Edition (WASI-2) (Wechsler, 1999) (Tscores: $\mathrm{ASD}=57.5 \pm 8.4, \mathrm{TD}=53.7 \pm 8.0)$. Individuals with ASDs were predominantly male $(\mathrm{ASD}=29, \mathrm{TD}=19)$, and had lower scores on the vocabulary subtest WASI-2 (T-scores: ASD $=53.6 \pm 10.6, \mathrm{TD}=61.9 \pm$ 8.7). Participants with ASDs were classified as high functioning based on WASI component scores. Participants with ASDs were diagnosed by research-reliable experimenters with either autistic disorder (25\%), Asperger's syndrome (66\%), or pervasive developmental disorder not otherwise specified (9\%) using Autism Diagnosis Observation Schedules (Lord et al., 2000) and/or Autism Diagnostic Interview-Revised (Lord et al., 1994), and a diagnosis by a practitioner familiar with ASD according to the DSM-IV (American Psychological Association, 2000). Individuals in the TD group had no diagnoses of ASD or any other psychiatric disorder. All participants had normal visual and auditory acuity as characterized in self-reports. Experimental protocols were approved by Vanderbilt University Medical Center's Institutional Review Board.

Stimuli. Visual stimuli were presented $60 \mathrm{~cm}$ from participants, and auditory stimuli were presented through centrally aligned speakers. Three categories of stimuli were presented (Fig. 1): simple flashes and beeps, dynamic handheld tools, and single syllable utterances ("ba" and "ga").

Procedures. Experiments took place in a dimly lit, sound-controlled room. Tasks and trials were randomized in all cases. For all tasks, participants were asked to fixate toward a cross and were actively monitored for compliance by a researcher sitting next to the participant. Participants completed an audiovisual simultaneity judgment (SJ) task with each stimulus type, a McGurk task, and auditory and visual temporal-order judgment tasks.

The audiovisual SJ tasks included presentations of a single stimulus type per run at parametrically varied stimulus onset asynchronies (SOAs), including audio-leading and visual-leading stimuli. For simple flashes and beeps, SOAs included $0, \pm 10, \pm 20, \pm 50, \pm 80$, and $\pm 100-$ $300 \mathrm{~ms}$ in $50 \mathrm{~ms}$ intervals. For tool stimuli, SOAs included 0 to $\pm 300 \mathrm{~ms}$ in $50 \mathrm{~ms}$ intervals. For speech stimuli, SOAs included 0 to $\pm 300 \mathrm{~ms}$ in 50

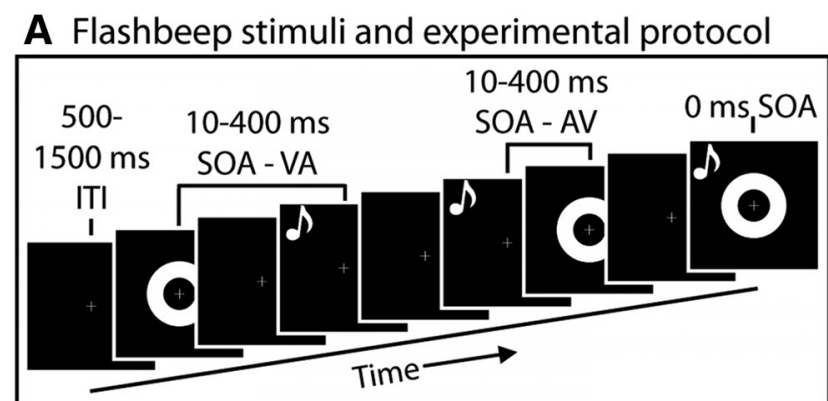

B Tool stimulus

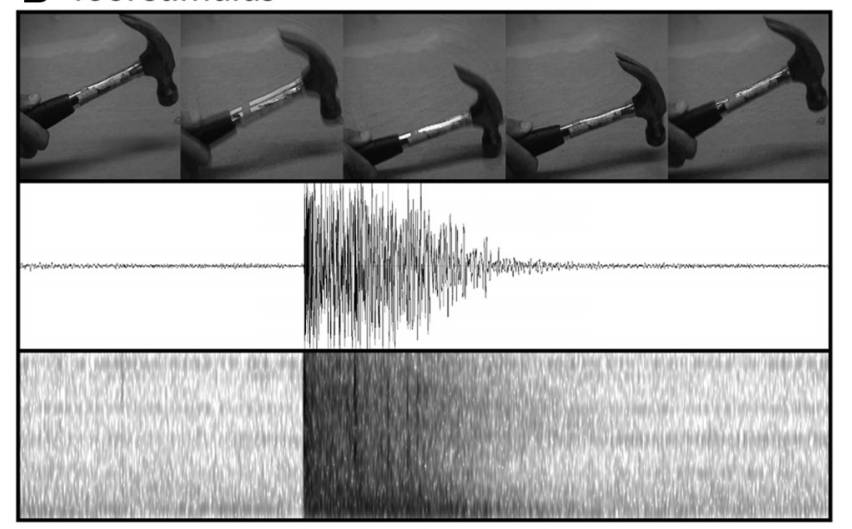

C /ga/ stimulus

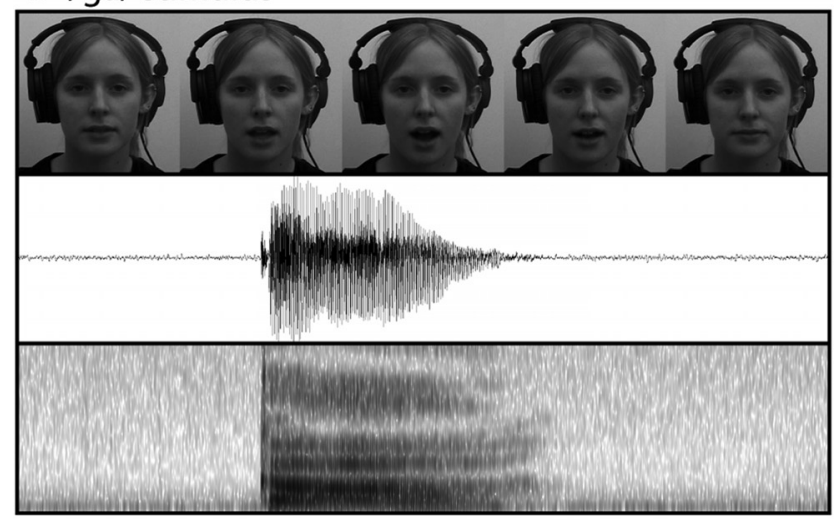

D /ba/stimulus

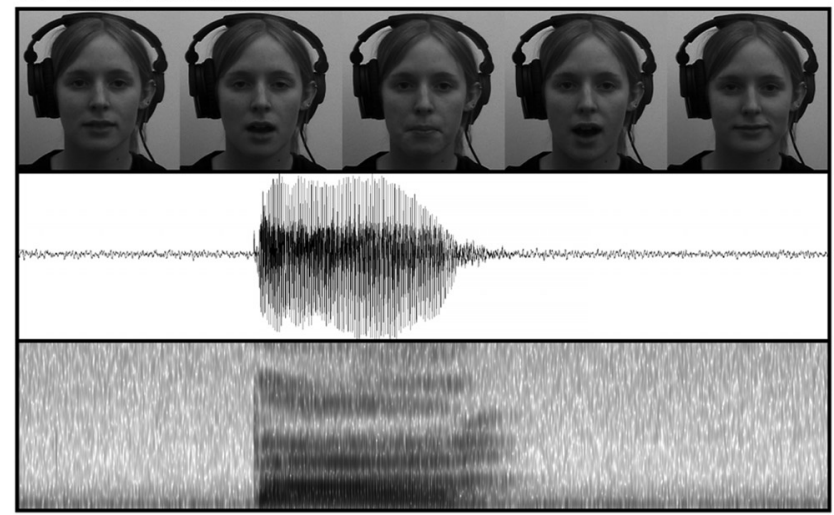

Figure 1. Stimulus types and trial format. $\boldsymbol{A}$, Trial format. $\boldsymbol{B}$, Tool stimuli. $\boldsymbol{C}, \boldsymbol{D}$, Speech stimuli. $\boldsymbol{B}-\boldsymbol{D}$, top to bottom, Individual frames from dynamic visual stimuli, the auditory waveform, and the auditory spectrogram. Trials began with a $500-1500 \mathrm{~ms}$ intertrial interval, followed by a stimulus presentation. After the stimulus presentation, a response screen appeared, and the next trail began after participants responded. 

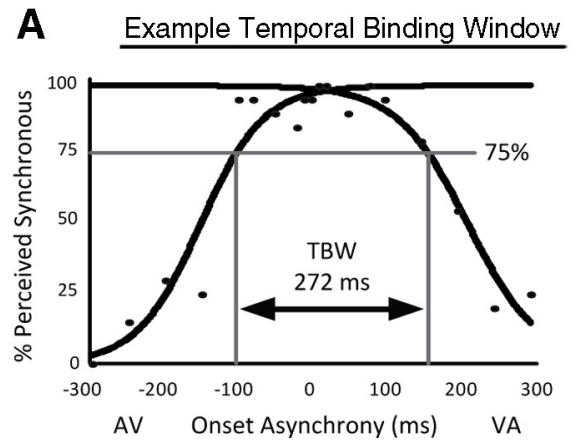

C Auditory Temporal Order Judgements

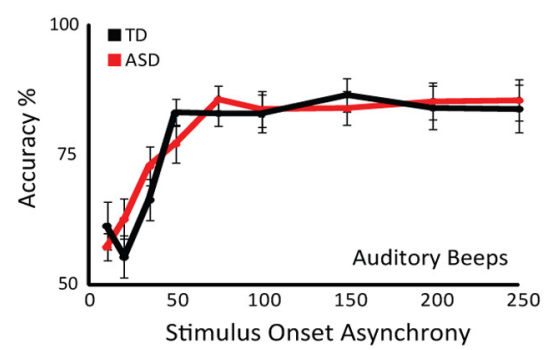

B Temporal Binding Window Widths

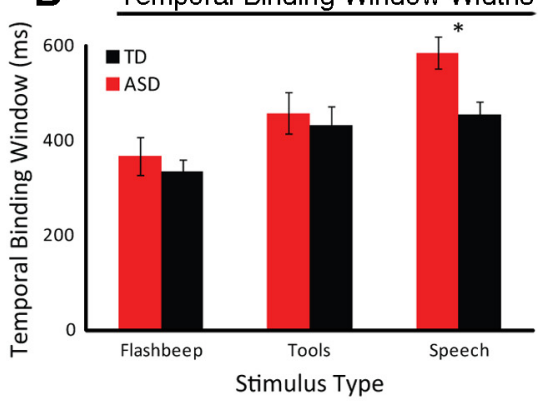

D Visual Temporal Order Judgements

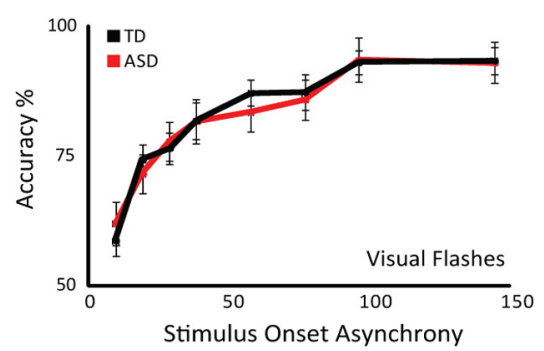

Figure 2. Mean individual multisensory temporal binding windows were calculated as depicted in $\boldsymbol{A}$ and averaged together for ASD (red) and TD (black) groups $(\boldsymbol{B})$. Significant differences across diagnostic groups were seen only with speech stimuli. No significant differences were seen between ASD and TD groups with either unisensory-auditory $(\boldsymbol{C})$ or unisensory-visual $(\boldsymbol{D})$ presentations. Error bars indicate SE. ${ }^{*} p<0.05$.

ms intervals and $\pm 400 \mathrm{~ms}$. Participants reported whether stimuli were temporally aligned and completed 20 trials per SOA.

For the McGurk task, trials included visual-only, auditory-only, and congruent audiovisual presentations of the phoneme "ba" or "ga," and the McGurk stimuli, a visual "ga" presented with an auditory "ba". All presentations were temporally synchronous. Participants reported what the speaker said by pressing one of four keys, "b," "g," "d," or “t." Twenty trials of each condition were presented.

Temporal-order judgment tasks were run with auditory and visual stimuli to control for unisensory temporal processing (SJ tasks were not used as the high acuity of unisensory simultaneity judgments often leads to a ceiling effect). Participants were presented with auditory or visual stimulus pairs in separate runs. Visual stimuli consisted of two white circles on a black background, above and below the fixation cross (duration $=10 \mathrm{~ms})$. Auditory stimuli consisted of a high- and low-pitch (100 and $500 \mathrm{~Hz}$ ) pair of beeps. Participants reported which came first. Unisensory SOAs ranged from 10 to $150 \mathrm{~ms}$ for visual stimuli and 10-250 ms for auditory stimuli.

Participants completed a two-subtest evaluation of the WASI-2, including the vocabulary and matrix reasoning subtests (Wechsler, 1999), a well-established measure of intelligence that has been commonly used with clinical and nonclinical samples.

Analysis. Mean responses from SJ tasks were used to calculate a temporal binding window (TBW) for each subject and stimulus category. Two psychometric sigmoid functions were fit to rates of perceived synchrony across SOAs: one to the audio-first (left) presentations and a second to the visual-first presentations (right). To account for non-zero points of subjective simultaneity, the SOA at which these two sigmoid functions crossed was extracted. If this point was greater or less than the next closest data point, two new sigmoid functions were fit splitting the data at the SOA at which the original sigmoid functions crossed. This process was continued in an iterative manner until the SOA at which best-fit sigmoid functions crossed fell between the two data points at which the data were split (Stevenson and Wallace, 2013).

Individuals' strength of McGurk effect was calculated as the percentage of multisensory trials in which he/she reported the fused (i.e.,
McGurk) percept relative to the percentage of trials in which they perceived an auditory-only "ba" or a visual-only "ga" as "da" or "tha," or: $\mathrm{p}($ AV McGurk $) \times[1-\mathrm{p}($ Unisensory “da” $)]$

where $\mathrm{p}$ (AV McGurk) is the individual's rate of McGurk percepts with audiovisual McGurk stimuli, and $\mathrm{p}$ (Unisensory " $\mathrm{da}$ ") is the rate at which the individual reported perceiving "da" with unisensory "ba" and "ga" stimuli (Fig. 1B).

\section{Results}

Multisensory temporal function differs in participants with ASD for speech-related stimuli

Multisensory temporal acuity was calculated for ASD and TD participants via the TBW using simple (flashes and beeps), complex non-speech (dynamic tools), and speech audiovisual stimuli. A $2 \times 3$ ANOVA revealed a significant main effect of stimulus type $\left(F_{(63,1)}=80.2, p<0.001\right.$, partial $\left.\eta^{2}=0.56\right)$, a marginal main effect of diagnosis $\left(F_{(63,1)}=3.26, p=0.076\right.$, partial $\left.\eta^{2}=0.05\right)$, and a significant interaction $\left(F_{(63,1)}=5.22, p=0.026\right.$, partial $\left.\eta^{2}=0.08\right)$. Subsequent to the significant interaction effect, follow-up $t$ tests revealed that individuals with ASDs showed decreased multisensory temporal acuity compared with TD individuals with speech stimuli $\left(t_{(31)}=3.49, p<0.05, d=0.83\right)$. In contrast, no difference was found with simple $\left(t_{(31)}=1.25, p=0.22, d=0.31\right)$ or complex $\left(_{(31)}=0.98, p=0.34, d=0.25\right)$ non-speech stimuli (Fig. 2).

\section{The McGurk effect reveals weaknesses in multisensory binding in ASD}

Individuals' rates of responses to the McGurk effect (a proxy measure for binding strength) were calculated. The pattern of responses to incongruent audiovisual (i.e., McGurk) stimuli differed between the ASD and TD groups (Fig. 3A). Individuals with ASDs were less likely to report the bound percepts (61\% vs 79\%; $\left.t_{(31)}=2.17, p<0.05, d=0.54\right)$ and more likely to report the auditory token $\left(33 \%\right.$ vs $\left.13 \% ; t_{(31)}=2.96, p<0.05, d=0.74\right)$ relative to the TD group. There was no difference between the report of the visual "ga" percept between the ASD and TD groups $\left(6 \%\right.$ vs $\left.9 \% ; t_{(31)}=0.74, p=0.46, d=0.19\right)$. Additionally, participants' perceptions with visual-only, auditory-only, and congruent multisensory were measured. No significant differences between groups were seen on these control conditions, including to audiovisual "ba" $\left(t_{(31)}=0.74, p=0.52, d=0.19\right)$ and "ga" $\left(t_{(31)}=0.69, p=0.41, d=0.17\right)$ presentations, to auditory-only "ba" $\left(t_{(31)}=0.02, p=0.99, d=0.01\right)$ and "ga" $\left(t_{(31)}=0.76\right.$, $p=0.31, d=0.19)$ presentations, and to visual-only "ba" $\left(t_{(31)}=\right.$ $0.82, p=0.23, d=0.21)$ and "ga" $\left(t_{(31)}=0.86, p=0.18, d=0.22\right)$ presentations (Fig. $3 B-D$ ). The lack of difference in the visual-only control conditions between-group differences suggests that differences in multisensory binding of speech signals are not attributable to face-processing or lip-reading differences between groups.

\section{Multisensory temporal function and the magnitude of the McGurk effect are strongly related in ASD}

The relationships between individuals' TBWs and their rates of McGurk perceptions were analyzed. The ASD group showed sig- 
nificant correlations between the strength of the McGurk effect and the width of the TBW measured using the flash-beep $(r=$ $0.46, p<0.05)$, tool $(r=0.51, p<0.03)$, and speech $(r=0.67, p<0.001)$ stimuli, with the strength of the correlation increasing systematically from simple through speech stimuli (Fig. 4B). In contrast, the TD group showed no such correlations when using flash-beep $(r=$ $0.04)$, tool $(r=0.04)$, or speech $(r=0.16)$ stimuli (Fig. 4B).

\section{Differences in multisensory temporal} function cannot be attributed to unisensory performance, sex, or vocabulary

Participants were tested on temporally based, unisensory control tasks. Performance on these visual- and auditory-only tasks failed to reveal any differences between the groups ( $\alpha=0.05$; Fig. 2). ANOVAs confirmed that sex showed no significant relation to TBWs associated with flashbeep $\left(F_{(63,1)}=0.44\right)$, tool $\left(F_{(63,1)}=\right.$ $2.24)$, or speech $\left(F_{(63,1)}=0.42\right)$ stimuli, or with McGurk effect scores $\left(F_{(63,1)}=0.17\right)$. Scores on the vocabulary component of the WASI were incorporated as covariates into the ANOVAs comparing the TBWs and McGurk effect rates across diagnostic groups. Vocabulary scores had no significant effect on TBWs with flashbeep $\left(F_{(63,1)}=0.08\right)$, tool $\left(F_{(63,1)}=0.81\right)$, or speech stimuli $\left(F_{(63,1)}=1.27\right)$, or on McGurk scores $\left(F_{(63,1)}=0.37\right)$.

\section{Discussion}

One of the core impairments in autism is a deficit in communicative ability, including speech perception and comprehension. Additionally, ASD has been linked to alterations in sensory processing, with an emphasis on the temporal aspects of such processing and a growing interest in understanding how changes in sensory function may result in changes in higher-order processes. The data presented here serve to bridge between these observations by showing strong links between multisensory temporal function and speech perception in ASDs. Specifically, the poorer an individual's temporal acuity across vision and audition (i.e., the larger their temporal binding window) even with simple flashes and beeps, the weaker their ability to bind auditory and visual speech to create a robust McGurk percept. The higher an individual's multisensory temporal acuity, the more reliable perceived synchrony is as a predictor of whether or not two sensory signals originated from a single external event as synchronous and should thus be perceptually bound. When an individual has low multisensory temporal acuity, the reliability of temporal information as a cue to determine which sensory signals should
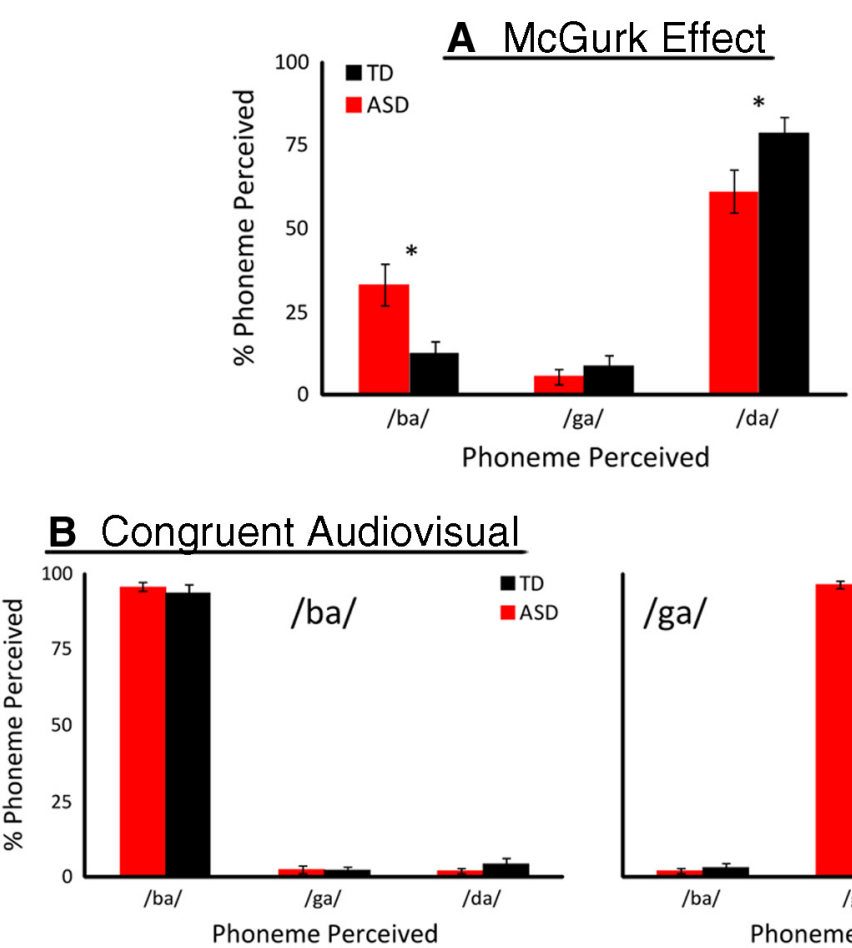

\section{Unisensory Auditory}
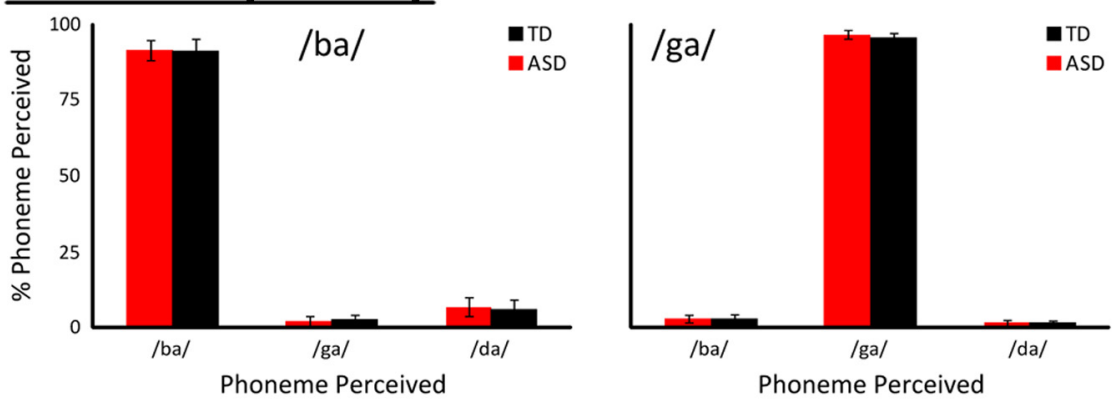

\section{Unisensory Visual}
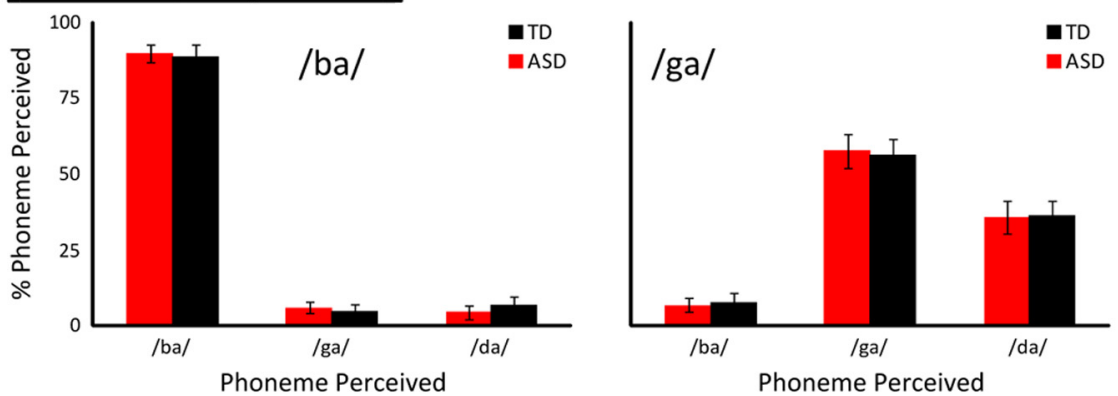

Figure 3. Rates of perceived syllables with audiovisual, incongruent McGurk trials are depicted for the ASD (red) and TD (black) groups in A. Individuals with ASD perceived the bound "da" percept less frequently and the auditory "ba" percept more frequently than TD controls. No significant differences were found in congruent audiovisual (B), unisensory-auditory $(\boldsymbol{C})$, or unisensory-visual (D) presentations. Error bars indicate SE. ${ }^{*} p<0.05$.

be integrated is greatly reduced, resulting in less efficient and weaker perceptual binding overall.

Multisensory temporal processing was indexed via the TBW, a probabilistic construct measuring the epoch of time within which multisensory inputs interact and are highly likely to be perceptually bound. The narrower the TBW, the more acute an individual's temporal perception in binding elements across audition and vision. Rather than being a static construct, the TBW appears to 
$\underline{\text { ATemporal Processing } x \text { Speech Integration in ASD }}$

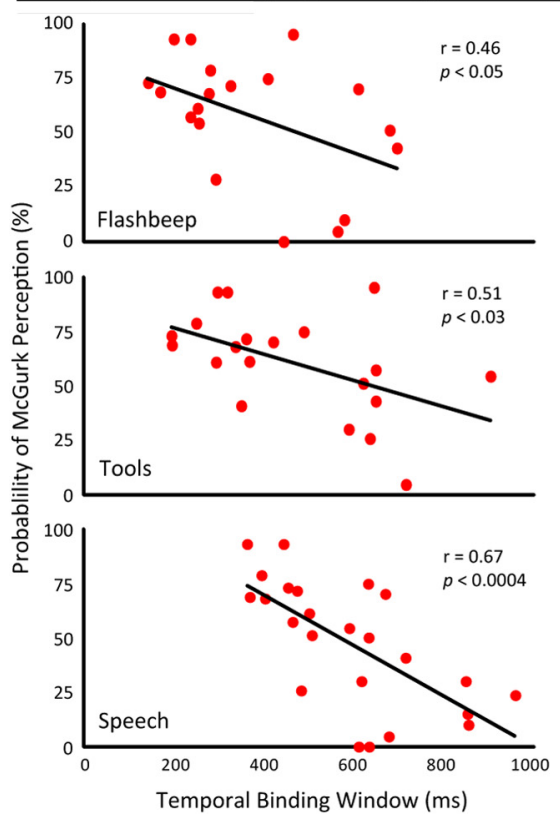

BTemporal Processing x Speech Integration inTD

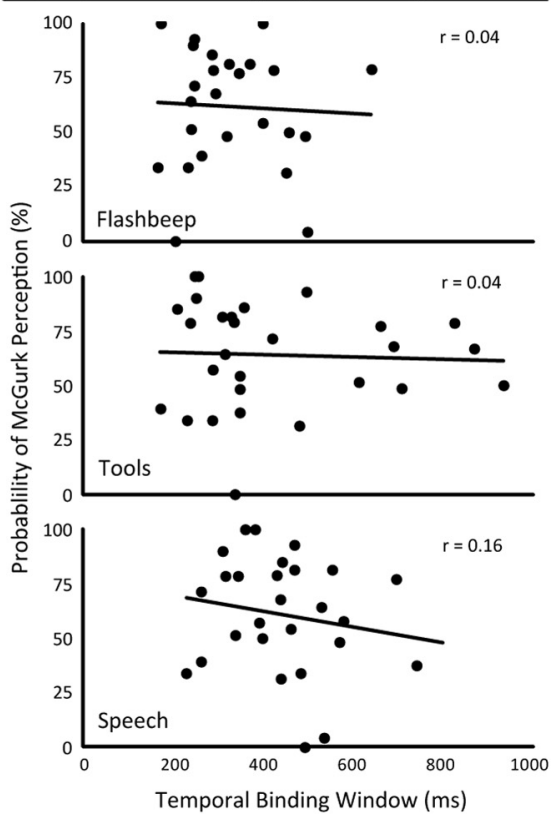

audiovisual speech integration (Stevenson et al., 2012). This would suggest that atypical multisensory temporal processing in ASDs may be associated with concurrent impairments in audiovisual speech perception that extend beyond the simple characterization of the window. To test this, we also measured the ability of individuals with and without ASDs to integrate audiovisual speech into a single perceptual gestalt, using the McGurk effect. Individuals with ASDs were less likely to report McGurk fusions, a finding that has been reported in some prior work in autism (Smith and Bennetto, 2007; Mongillo et al., 2008). As opposed to reporting the fused percept, participants with ASDs more often reported the auditory token (compared with TD individuals), a finding also consistent with prior work (Mongillo et al., 2008; Irwin et al., 2011). It is important to note here that differences in illusory McGurk percepts between individuals with and without ASDs could be attributable to differences is either lip reading or impaired face processing, characteristics that have been associated with

be highly dynamic, being malleable dependent upon prior history and task demands (Powers et al., 2009; Mégevand et al., 2013). In the current study, the size of the TBW for both the ASD and TD groups was found to also be dependent upon the complexity of the stimuli that were used in the assessment, with the largest windows being seen for stimuli of highest complexity (i.e., speech). A preferential enlargement of the TBW was seen in the ASD group, and this difference appeared to be specific for speech stimuli. However, we did find a marginal effect of diagnosis collapsed across stimulus types, in line with previous research suggesting that individuals with ASD may also show temporal processing deficits with simpler stimuli (Foss-Feig et al., 2010; Kwakye et al., 2011; de Boer-Schellekens et al., 2013).

A wider TBW with speech-related stimuli fits with previous lines of work evidencing differential narrowing in the development of the TBW dependent upon the nature of the stimuli used (Hillock et al., 2011), as well as with previous evidence that the synchrony-detection impairments seen in ASD are specific to speech (Bebko et al., 2006). A plausible explanation of the differential width of the TBW based on stimulus type is that more complex stimuli may require additional and/or more variable processing within modality before, or concurrent with, integration across modalities; thus, the multisensory process may be optimized with more tolerance for temporal offset as stimulus complexity increases. Speech-specific differences in multisensory temporal binding provide evidence that speech-processing times may be extended in ASDs (Oram Cardy et al., 2005), or there may be increased variance of the within-modality processing time for speech stimuli in either or both modalities. These findings are consistent with the temporal binding hypothesis of autism, which posits that the impairments in integrating information in ASDs result from decreased neural synchronization across neural networks (Brock et al., 2002).

Recent work in typical populations has illustrated that individuals exhibiting more acute temporal multisensory processing (i.e., narrower multisensory TBWs) also exhibit increased rates of
ASDs (Gelder et al., 1991; Deruelle et al., 2004; Smith and Bennetto, 2007). However, in the current data, such differences would also be seen in visual-only conditions in which the participants are required to both attend faces and read lips, and results from these conditions showed striking similarities across diagnostic groups, reinforcing that the differences are likely the result of atypical multisensory processing.

Perhaps of greatest interest here was the strong association in ASD between individuals' TBWs and the strength of their McGurk effect. Simply stated, those with wider TBWs were less likely to perceptually bind audiovisual speech. That this relationship between audiovisual speech binding and temporal processing was indexed using even very simple stimuli (i.e., flashes and beeps) suggests that impairments in low-level temporal sensory processing may possibly cascade into higher-order domains, such as speech perception in ASDs. Indeed, future longitudinal work tracking the developmental trajectory of these features needs to extend these relationships to those more tightly tied to functional language scores.

The link between temporal processing and audiovisual speech integration can also be seen in their underlying neural correlates, where similar substrates have been implicated in the processing of audiovisual stimuli and the fine temporal structure of sensory stimuli (Miller and D'Esposito, 2005; Stevenson et al., 2010), as well as in the integration of audiovisual speech stimuli (Stevenson et al., 2011; Nath and Beauchamp, 2012). These studies have converged on the cortex of the posterior superior temporal sulcus (pSTS) as a major node in these processes. Furthermore, the pSTS has been shown to exhibit both anatomical (e.g., Levitt et al., 2003) and functional (e.g., Pelphrey and Carter, 2008) differences in individuals with ASDs, reinforcing its role as a major hub for the integration of audiovisual speech stimuli.

This strong relationship between low-level multisensory temporal function and speech perception in ASDs also provides a possible avenue for intervention, as perceptual training paradigms have been shown to be effective in narrowing the TBW 
(Powers et al., 2009; Stevenson et al., 2013). Most encouragingly, these prior studies, conducted in typical adults, showed that those with the widest TBWs were those to exhibit the greatest narrowing after training. Reinforcing the role of the pSTS in these processes, a neuroimaging follow-up to these training studies showed the neural correlates of these effects to be centered on a network of cortical regions in which pSTS was the locus of the training-related changes (Powers et al., 2012).

The current study is the first to establish significant links between aspects of sensory function, specifically multisensory temporal function, and the higher-order domain of speech perception, long known to represent a core weakness in autism. Such a relationship makes a great deal of intuitive sense, in that sensory and multisensory representations make up the "building blocks" upon which higher-order representations, such as those responsible for communication and social function, are built. However, despite this intuition, and the wealth of evidence for sensory, communication, and social dysfunction on autism, no work before this has empirically explored these intersections, intersections likely to reveal important insights into the autistic brain and how sensory plasticity may be engaged to improve functional outcomes.

These results suggest prospective avenues for future work. First, these findings may be applicable in other populations that show atypical temporal processing and multisensory integration, including developmental dyslexia (Hairston et al., 2005; BastienToniazzo et al., 2009) and schizophrenia (Davalos et al., 2002; de Gelder et al., 2003). Second, the link between multisensory temporal processing and multisensory perceptual binding in TD can be seen across but not within studies. Uncorrelated TBWs and McGurk scores in the TD group were surprising given similar developmental time courses and that such a correlation has been found in TD adults (Stevenson et al., 2012). Third, to our knowledge, development of multisensory temporal processing in ASDs has not been investigated in any way, despite a growing literature on multisensory temporal deficits in ASDs. Studies examining the developmental trajectory of multisensory temporal processing, specifically using longitudinal designs, have the potential to make a tremendous impact in the field and could identify key ages at which sensory-based remediation approaches may be most successful. Finally, although temporal cues are one powerful factor in multisensory integration and binding, other stimulusrelated factors also play an integral role in the construction of an integrated perceptual gestalt. Recent work has focused on one of these factors, stimulus effectiveness (as evaluated by signal-tonoise ratio), and has shown there to be interesting differences between ASD and TD children in how multisensory processing changes as a function of changes in effectiveness (Foxe et al., 2013).

\section{References}

American Psychological Association (2000) Diagnostic and statistical manual of mental disorders-IV-TR. Washington, DC: American Psychological Association.

Bastien-Toniazzo M, Stroumza A, Cavé C (2009) Audio-visual perception and integration in developmental dyslexia: an exploratory study using the McGurk effect. Curr Psychol Lett 25.

Bebko JM, Weiss JA, Demark JL, Gomez P (2006) Discrimination of temporal synchrony in intermodal events by children with autism and children with developmental disabilities without autism. J Child Psychol Psychiatry 47:88-98. CrossRef Medline

Bertone A, Mottron L, Jelenic P, Faubert J (2003) Motion perception in autism: a "complex" issue. J Cogn Neurosci 15:218-225. CrossRef Medline

Brandwein AB, Foxe JJ, Butler JS, Russo NN, Altschuler TS, Gomes H, Mol- holm S (2013) The development of multisensory integration in highfunctioning autism: high-density electrical mapping and psychophysical measures reveal impairments in the processing of audiovisual inputs. Cereb Cortex 23:1329-1341. CrossRef Medline

Brock J, Brown CC, Boucher J, Rippon G (2002) The temporal binding deficit hypothesis of autism. Dev Psychopathol 14:209-224. Medline

Davalos DB, Kisley MA, Ross RG (2002) Deficits in auditory and visual temporal perception in schizophrenia. Cogn Neuropsychiatry 7:273-282. CrossRef Medline

de Boer-Schellekens L, Eussen M, Vroomen J (2013) Diminished sensitivity of audiovisual temporal order in autism spectrum disorder. Front Integr Neurosci 7:8. CrossRef Medline

de Gelder B, Vroomen J, Annen L, Masthof E, Hodiamont P (2003) Audiovisual integration in schizophrenia. Schizophr Res 59:211-218. CrossRef Medline

Deruelle C, Rondan C, Gepner B, Tardif C (2004) Spatial frequency and face processing in children with autism and Asperger syndrome. J Autism Dev Disord 34:199-210. CrossRef Medline

Foss-Feig JH, Kwakye LD, Cascio CJ, Burnette CP, Kadivar H, Stone WL, Wallace MT (2010) An extended multisensory temporal binding window in autism spectrum disorders. Exp Brain Res 203:381-389. CrossRef Medline

Foxe JJ, Molholm S, Del Bene VA, Frey HP, Russo NN, Blanco D, SaintAmour D, Ross LA (2013) Severe multisensory speech integration deficits in high-functioning school-aged children with autism spectrum disorder (ASD) and their resolution during early adolescence. Cereb Cortex. Advance online publication. Retrieved. doi:

Frith U, Happe F (1994) Autism: beyond "theory of mind." Cognition 50: 115-132. CrossRef

Gelder B, Vroomen J, van der Heide L (1991) Face recognition and lipreading in autism. Eur J Cogn Psychol 3:69-86. CrossRef

Hairston WD, Burdette JH, Flowers DL, Wood FB, Wallace MT (2005) Altered temporal profile of visual-auditory multisensory interactions in dyslexia. Exp Brain Res 166:474-480. CrossRef Medline

Hillock AR, Powers AR, Wallace MT (2011) Binding of sights and sounds: age-related changes in multisensory temporal processing. Neuropsychologia 49:461-467. CrossRef Medline

Irwin JR, Tornatore LA, Brancazio L, Whalen DH (2011) Can children with autism spectrum disorders "hear" a speaking face? Child Dev 82:13971403. CrossRef Medline

Kanner L (1943) Autistic disturbances of affective contact. Nervous Child 2:217-250.

Kwakye LD, Foss-Feig JH, Cascio CJ, Stone WL, Wallace MT (2011) Altered auditory and multisensory temporal processing in autism spectrum disorders. Front Integr Neurosci 4:129. CrossRef Medline

Levitt JG, Blanton RE, Smalley S, Thompson PM, Guthrie D, McCracken JT, Sadoun T, Heinichen L, Toga AW (2003) Cortical sulcal maps in autism. Cereb Cortex 13:728-735. CrossRef Medline

Lord C, Rutter M, Le Couteur A (1994) Autism Diagnostic Interview-Revised: a revised version of a diagnostic interview for caregivers of individuals with possible pervasive developmental disorders. J Autism Dev Disord 24:659-685. CrossRef Medline

Lord C, Risi S, Lambrecht L, Cook EH Jr, Leventhal BL, DiLavore PC, Pickles A, Rutter M (2000) The autism diagnostic observation schedule-generic: a standard measure of social and communication deficits associated with the spectrum of autism. J Autism Dev Disord 30:205-223. CrossRef Medline

Marco EJ, Hinkley LB, Hill SS, Nagarajan SS (2011) Sensory processing in autism: a review of neurophysiologic findings. Pediatr Res 69:48R-54R. CrossRef Medline

McGurk H, MacDonald J (1976) Hearing lips and seeing voices. Nature 264:746-748. CrossRef Medline

Mégevand P, Molholm S, Nayak A, Foxe JJ (2013) Recalibration of the multisensory temporal window of integration results from changing task demands. PLoS One 8:e71608. CrossRef Medline

Miller LM, D'Esposito M (2005) Perceptual fusion and stimulus coincidence in the cross-modal integration of speech. J Neurosci 25:5884-5893. CrossRef Medline

Mongillo EA, Irwin JR, Whalen DH, Klaiman C, Carter AS, Schultz RT (2008) Audiovisual processing in children with and without autism spectrum disorders. J Autism Dev Disord 38:1349-1358. CrossRef Medline

Nath AR, Beauchamp MS (2012) A neural basis for interindividual differ- 
ences in the McGurk effect, a multisensory speech illusion. Neuroimage 59:781-787. CrossRef Medline

Oram Cardy JE, Flagg EJ, Roberts W, Brian J, Roberts TP (2005) Magnetoencephalography identifies rapid temporal processing deficit in autism and language impairment. Neuroreport 16:329-332. CrossRef Medline

Pelphrey KA, Carter EJ (2008) Charting the typical and atypical development of the social brain. Dev Psychopathol 20:1081-1102. CrossRef Medline

Powers AR 3rd, Hillock AR, Wallace MT (2009) Perceptual training narrows the temporal window of multisensory binding. J Neurosci 29:1226512274. CrossRef Medline

Powers AR 3rd, Hevey MA, Wallace MT (2012) Neural correlates of multisensory perceptual learning. J Neurosci 32:6263-6274. CrossRef Medline

Smith EG, Bennetto L (2007) Audiovisual speech integration and lipreading in autism. J Child Psychol Psychiatry 48:813-821. CrossRef Medline

Stevenson RA, Wallace MT (2013) Multisensory temporal integration: task and stimulus dependencies. Exp Brain Res 227:249-261. CrossRef Medline
Stevenson RA, Altieri NA, Kim S, Pisoni DB, James TW (2010) Neural processing of asynchronous audiovisual speech perception. Neuroimage 49: 3308-3318. CrossRef Medline

Stevenson RA, VanDerKlok RM, Pisoni DB, James TW (2011) Discrete neural substrates underlie complementary audiovisual speech integration processes. Neuroimage 55:1339-1345. CrossRef Medline

Stevenson RA, Zemtsov RK, Wallace MT (2012) Individual differences in the multisensory temporal binding window predict susceptibility to audiovisual illusions. J Exp Psychol Hum Percept Perform 38:1517-1529. CrossRef Medline

Stevenson RA, Wilson MM, Powers AR, Wallace MT (2013) The effects of visual training on multisensory temporal processing. Exp Brain Res 225: 479-489. CrossRef Medline

Sumby WH, Pollack I (1954) Visual contribution to speech intelligibility in noise. J Acoustical Soc Am 26:212-215. CrossRef

Wechsler D (1999) Wechsler Abbreviated Scale of Intelligence. San Antonio: Psychological Corporation. 